\title{
Polysèmes
}

Revue d'études intertextuelles et intermédiales

\section{« Terror has no diary » : Melmoth ou le récit éviscéré}

\section{Catherine Lanone}

\section{(2) OpenEdition}

Journals

Édition électronique

URL : http://journals.openedition.org/polysemes/1647

DOI : 10.4000/polysemes. 1647

ISSN : 2496-4212

Éditeur

SAIT

Édition imprimée

Date de publication : 1 janvier 2005

Pagination : 121-135

ISSN : 0999-4203

\section{Référence électronique}

Catherine Lanone, « «Terror has no diary »: Melmoth ou le récit éviscéré », Polysèmes [En ligne],

7 | 2005, mis en ligne le 01 janvier 2005, consulté le 30 avril 2019. URL : http://

journals.openedition.org/polysemes/1647 ; DOI : 10.4000/polysemes.1647

Ce document a été généré automatiquement le 30 avril 2019.

Polysèmes 


\title{
"Terror has no diary $»^{1}:$ Melmoth ou le récit éviscéré
}

\author{
Catherine Lanone
}

Paru en 1820, Melmoth the Wanderer de R.C. Maturin est souvent considéré comme le dernier grand roman noir, avec son protagoniste archétypal, maudit, mais qui paradoxalement «sert indirectement les desseins du ciel $»^{2}$, selon le mot d'Hubert Teyssandier. C'est l'outrance qui donne au roman la tonalité si sombre et si particulière qui séduisait Baudelaire. Pour André Breton, l'écriture se nourrit à même l'incandescence de la violence, comme dictée par l'inconscient ; Melmoth est la comète du mal traversant l'ogive gothique, se consumant pour suspendre «l'interminable pluie de cendres » d'un genre qui se délite ${ }^{3}$. L'excès fait violence au texte même, comme pour dépasser la figuration, c'est-à-dire procéder non vers l'abstraction mais vers la Figure, ou la sensation deleuzienne: "La Figure, c'est la forme sensible rapportée à la sensation; elle agit immédiatement sur le système nerveux, qui est de la chair $»^{4}$. Ainsi, Melmoth va au-delà des paradigmes gothiques sur lesquels le roman s'appuie, pour proposer une véritable logique de la sensation, qui problématise le fonctionnement du texte lui-même et dévie l'interprétation de la violence.

\section{Les paradigmes de la violence gothique}

2 Puisque, selon Deleuze, à « la violence du représenté (le sensationnel, le cliché) s'oppose la violence de la sensation $»^{5}$, c'est par le cliché qu'il faut commencer, avant d'aborder le véritable frémissement du texte. Le scénario de Melmoth décline allègrement les lieux communs du genre, des souterrains inquiétants à la plongée vers le passé et la séduction de la pure jeune fille. David Punter rappelle que l'essence du gothique, c'est la terreur'. Maturin sait que cette magie noire lui convient, comme il l'écrit à Walter Scott : «I wish they would let me do what I am good for, sit down by my magic cauldron, mix my dark ingredients, see the bubbles work, and the spirits rise ${ }^{7}$. Il se propose cette fois de surpasser Hérode ${ }^{8}$ et les maîtres gothiques, tout un programme. Comme dans The Monk de M.G. Lewis, le scénario repose sur le pacte avec le diable, mais ici ce pacte a déjà eu lieu, et 
depuis longtemps. Mi-Faust mi-Méphistophélès, Melmoth doit séduire une âme à son tour, et pour mieux tenter apparaît toujours à un moment de souffrance paroxystique.

3 La première figure de la violence, dans le gothique, c'est le labyrinthe architectural, le non-lieu angoissant, fragmenté, déjà une forme d'oppression qui connote le vide. Le mariage de Melmoth et d'Immalee est célébré par une figure de l'ombre - dont la main glacée suggère celui que Lacassin surnomme le grand maître de cérémonies ${ }^{9}$, le diable en personne - dans une chapelle déserte à la Friedrich, avec ses pans de murs en ruine, vasques vides ou rosaces et crucifix brisés. Mais chez Friedrich la toile s'ouvre à la lueur pâle de la transcendance romantique, tandis que chez Maturin la scène reste dans la pénombre. L'abbaye signe le morcellement de la personnalité d'Immalee, la ruine devenant ce « lieu d'écrasement du sens, lieu poignant qui neutralise la distinction passé/ présent/avenir, lieu de l'indifférencié absolu et de l'insignifiant, la mort $»^{10}$, pour reprendre la définition de Philippe Hamon.

Outre la ruine, le roman joue aussi sur l'asile de fous où l'on enferme Stanton alors qu'il jouit de toute sa raison, les cellules de l'Inquisition, ou les souterrains du couvent, autant de clichés du catholicisme pervers traditionnellement dépeint par le gothique. La cellule nue de Monçada, comme la geôle où les serpents suintent des murs - version magnifiée des vers venus ronger le corps dans le tombeau -, le souterrain où s'éteint la lampe, suggèrent la violence faite à l'individu, surtout lorsque la métaphore confère au lieu suintant une visagéité monstrueuse : « the black and dripping walls, and the low arches of the vault, that seemed to lower over me like the frown of an eternal hostility (214). Selon Francis Lacassin, souffrir n'est rien si l'on ne souffre pas dans l'obscurité. C'est alors qu'intervient l'une des deux occurrences de cette expression prégnante, «terror has no diary ", qui est modulée ici par "the noctuary of terror » (213). Plus que la description, c'est cette torsion faite à la langue qui figure l'oppression, et confère plus d'originalité à la topographie de Maturin.

5 À la carte abjecte, correspond la convulsion du sujet soumis à la souffrance, sujet qui se délite, se défait, à mesure que sont violentées les frontières du corps propre. Yves-Alain Michaux définit la violence par le désordre; citant Freund, il ajoute qu'elle n'est pas, comme on le croit généralement, un abus de la force, mais un «abus de la puissance " ${ }^{11}$. C'est sur ce rapport de forces que jouent l'Inquisition et la question, régulant cette violence du langage que Lecercle décrit en citant Canetti, «Questionner, c'est entrer par force $»^{12}$. La parole est extirpée, et régulée; dans les cachots de l'Inquisition, le prisonnier doit parler à voix basse. Maturin s'intéresse en fait moins à la torture physique qu'au système sémiotique hypocrite qui constitue une formidable machine de guerre. Ainsi, la métaphore redevient littérale, pour jouer sur l'affect. Franchir la limite symbolique, c'est enfreindre les seuils de l'espace et du corps. Lorsque Monçada se refuse à prononcer ses vœux, il est sommé de marcher sur le corps de sa mère pour sortir. Plus tard, au couvent, excommunier, c'est rompre toute communication possible. Le repas pris en commun est remplacé par l'exclusion d'un corps rejeté vers le déchet: Monçada ne peut même plus pénétrer dans le réfectoire et doit se contenter de restes immondes connotant le cadavre, puisque s'y mêlent «ashes, hair and dust » (168). Sur les murs de sa cellule entièrement dépouillée apparaissent les hiéroglyphes de la violence, flammes et démons : « Desperate with horror, I rushed against the walls, and found that what I touched was cold. My recollection returned, and I comprehended that these were hideous figures scrawled in phosphorus, to terrify me » (168). 
6 L'épisode accentue la dimension onirique du mal. L'imagination contribue toujours à dilater, défigurer le présent pour y introduire la souffrance à venir. Attendre le châtiment, c'est déjà souffrir ; le rêve accentue l'anticipation néfaste. La torture mentale est raffinée, la forme la plus subtile étant l'espoir. Ainsi Monçada s'enfuit en compagnie d'un parricide, rampe dans un boyau mortifère et suintant, franchit le mur du couvent après vingt-quatre heures de ténèbres affamées; son frère qui l'attend s'écrie «il est sauvé ! » puis s'écroule dans une mare de sang. Monçada s'évanouit et se réveille dans le cachot de l'Inquisition.

7 Une figure significative de la violence, ici, ce serait donc son aspect théâtral, spectaculaire, relevant d'une mise en scène du rapport de pouvoir, avec ses accessoires factices et ses corps bien réels, ses stratagèmes et ses coups de théâtre.

8 Or, cette spectacularisation de la violence, c'est ce qui permet aussi de franchir la limite entre le corps de désir et le corps abject, entre la chair vive et la viande morte, entre l'amour et la consommation cannibale. C'est cette rupture du tabou qui se joue à diverses reprises, selon des relais symboliques limpides ${ }^{13}$.

Dans le récit du parricide, la porte fait écran, mais la dévoration se met en scène sur le mode du fantasme. Le parricide se repait des cris des deux amants enterrés vifs, de leurs hurlements de faim, puis de haine succédant à l'amour. Le zeugme rend compte du transfert suggérant la jouissance de l'ogre : « while I was eating, I actually lived on the famine that was devouring them»(235), "They were rapidly becoming objects of hostility to each other,-oh what a feast to me! »(236). La porte occulte, pour mieux représenter cette dévoration que frôle le texte; et si le parricide dévore sur le mode métaphorique, le jeune homme, lui, tente de manger sa compagne, même si l'on ne trouvera, une fois la porte ouverte, qu'une simple trace de morsure. Plus tard, lorsque la foule met en pièces le parricide, la transformation de la chair en viande s'achève. Les métaphores de corrida scandant le démembrement, jusqu'à ce qu'il ne reste plus qu'un cri, un visage décomposé en plaies, puis une masse abjecte, des lambeaux rouges qui n'ont plus rien d'humain :

Alors la bouche acquiert cette puissance d'illocalisation qui fait de toute viande une tête sans visage. Elle n'est plus un organe particulier, mais le trou par lequel le corps tout entier s'échappe, et par lequel descend la chair [...] Ce que Bacon appelle le Cri dans l'immense pitié qui entraîne la viande. ${ }^{14}$

10 On pourrait songer ici que cette violence s'inscrit dans la logique d'une forme de justice, celle du bouc émissaire mise en évidence par René Girard dans La Violence et le sacré. En effet, la scène se déroule lors d'une procession, rite catholique dégénéré, où l'immolation dionysiaque pourrait figurer la catharsis. La fête qui tourne mal où la victime arbitraire est déchirée à mains nues aurait alors une valeur sacrificielle : selon Girard, «sa mort violente procure à la foule l'exutoire dont elle a besoin pour retrouver le calme $»^{15}$.

11 Mais il faudrait pour cela croire à une logique de la rédemption, qui n'est au fond pas si différente du catholicisme exhibant le corps crucifié, figure de violence mutée en principe de rédemption. L'essentiel, dans le texte de Maturin, se joue en fait ailleurs.

\section{L'agencement paranoïaque du récit}

12 Ce que Maturin représente, c'est moins le récit linéaire d'une séduction maléfique, avec un ennemi sournois dont il faudrait triompher, qu'une économie de la violence 
généralisée. Le discours du mal se retourne vers l'humain. La violence qui se propage provient de l'homme, non du diable. À la jubilation cannibale du parricide, répond la métamorphose des amants, le passage de l'amour à la haine, et la violation du corps de l'amante. Les conditions extrêmes de violence corrompent toute valeur, tout sentiment. C'est en ce sens qu'il faut lire l'épisode central d'Immalee, assez long et lent, mais qui offre une méditation sur le mal. Immalee incarne la chute de l'innocence à l'expérience. C'est un thème banal, mais la vraie tentation qu'offre Melmoth à l'innocente sur son île déserte, c'est la connaissance du mal humain. À l'aide d'un télescope, il lui fait voir ce qu'adorent les hommes, autant d'édifices sacrificiels, décorés de fleurs et de bébés dans des cages. On pourrait lire là un discours manichéen sur les sauvages, tel qu'il ressurgira chez Ballantyne par exemple, si ce sacrifice n'était mis en parallèle avec l'éducation qu'Immalee retrouvée reçoit en Espagne. Si l'on sacrifiait les bébés sur la rive exotique, la jeune femme doit être mariée au plus offrant, de même que la mère de Monçada voulait vendre son fils au couvent pour se racheter. Il ne s'agit plus de salut, mais tout simplement de racheter les biens temporels et intemporels, en monnayant la souffrance d'autrui, et surtout de ses propres enfants. Aussi la flagellation par laquelle le Supérieur prétend expier la vocation forcée de Monçada devient-elle l'envers du pacte infernal: "Oh! how false is a treaty made with God, which we ratify with our own blood!»(106). Les italiques soulignent la perversion d'une mascarade qui couvre la vraie monnaie d'échange, la violence faite à Monçada.

Le parricide formule clairement l'économie abjecte qui sous-tend toutes les institutions, selon ce qu'il appelle "the theology of hostility : «In this flattering theology, your crimes become my virtues. I need not any of my own » (250). Chacun se lave dans la faute et le sang de l'autre, sous l'égide de l'Inquisition.

C'est alors que l'on perçoit la formidable torsion que fait subir Maturin au scénario programmatique du pacte avec le diable : le roman impose à tous ses personnages le sort de Job, mais la tentation n'est en fait orchestrée ni par Dieu ni par le diable ; la violence relève toujours de l'humain. Intolérance, avarice, cupidité sont les mobiles de la mort par la faim ou le feu, de l'enfermement dans la cellule d'aliénés ou la geôle de l'inquisition; Melmoth, en fait, tente de profiter de la situation qu'il trouve pour proposer son pacte, il ne la crée pas, il en est le spectateur plus que l'acteur. Lorsque la violence se griffonne au phosphore sur les murs, l'Enfer, c'est les autres.

Puisque le mal est humain, le scénario se déroute. Le vrai tentateur, c'est moins Melmoth, qui n'apparait au fond des cellules que pour prêcher sans succès l'horreur, c'est la figure dégradée, déchue du parricide, qui a symboliquement effacé l'ordre du Père, pour jouir du désordre souterrain, et tenter les amants, puis Monçada ; faire alliance avec le parricide, c'est emprunter la voie de la fuite pour se retrouver dans l'enfer d'une souffrance pire encore, et bien terrestre. Voilà qui nous rappelle le pacte toujours pipé avec le diable, mais ici il a été rogné, retaillé à mesure humaine.

16 Il faut alors poser le problème du regard que le texte nous oblige à porter sur ces figures de la violence qu'il paraissait étaler si complaisamment. Le texte met en scène ce regard, un peu à la façon dont Delacroix structure son tableau Sardanapale. Dans un style très différent, la scène exotique représente la mise à mort des femmes et des animaux de Sardanapale qui, vaincu, va se suicider. L'intérêt, c'est bien sûr la tension qui s'établit entre la débauche de violence au premier plan, en un flamboiement de tissus rouges qui supplée au sang invisible, et la pose alanguie de Sardanapale, qui est lui fort habillé, et semble prendre un intérêt lascif à la scène. Delacroix dramatise ici à la fois l'érotisme de 
la violence et le problème du regard porté sur elle. Le voyeurisme de Sardanapale tend un miroir au spectateur.

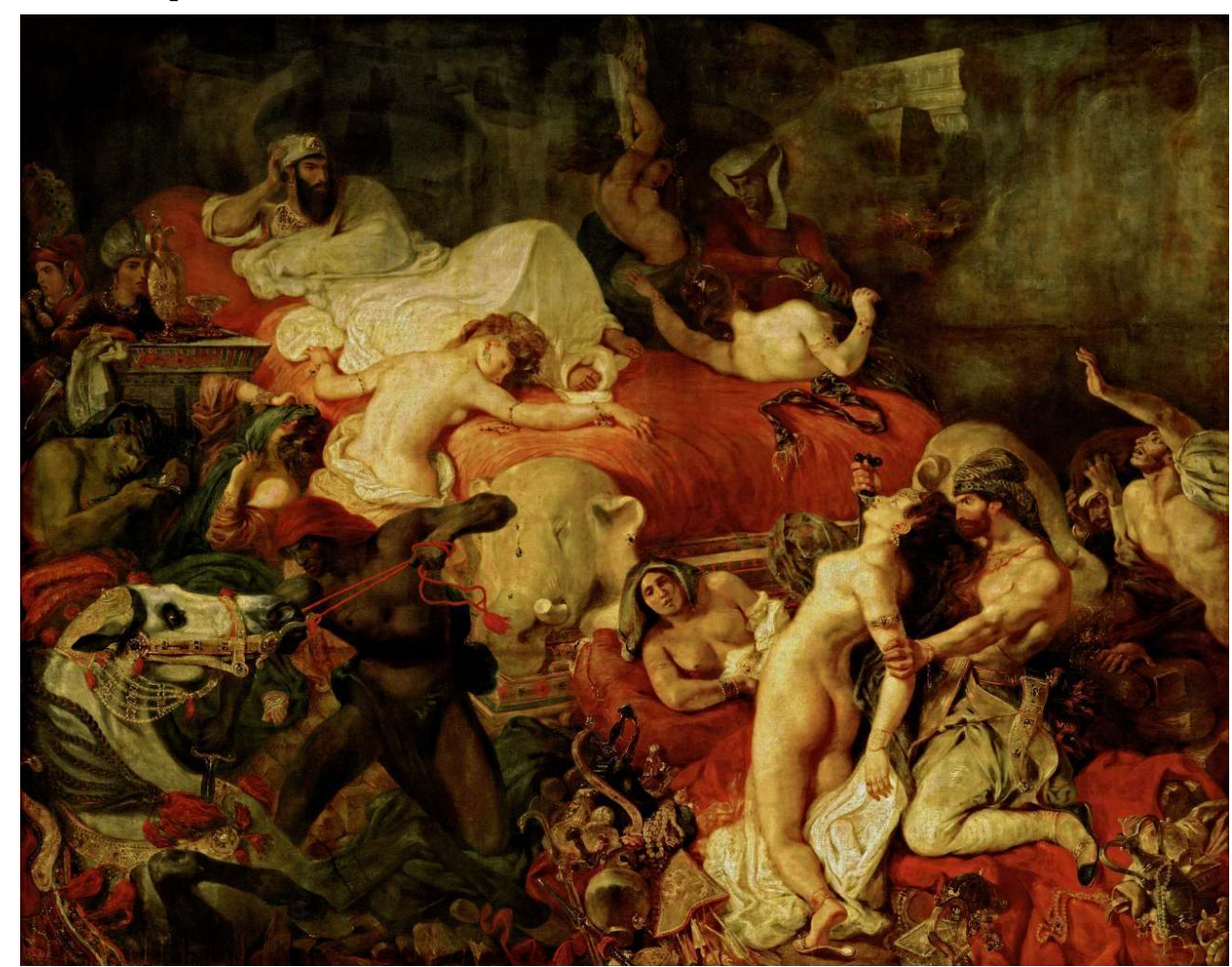

Eugène Delacroix, La Mort de Sardanapale (1827), huile sur toile, 3,92×4,96m, Musée du Louvre, Wikimedia Commons

\section{Le regard, ou la violence dé-figurée}

Or, comme le tableau de Delacroix, le texte de Melmoth vient questionner ostensiblement le regard porté sur la violence. Le genre est d'abord problématisé par des allusions métatextuelles ironiques, à Lewis, par exemple, ou aux topoï inévitables : «Romances have made your country, sir, familiar with tales of subterranean passages, and supernatural horrors » (212). Le voyeurisme du lecteur est confronté à la jouissance à la Sardanapale du parricide, et sa fameuse profession de foi :

It is actually possible to become amateurs in suffering. I have heard of men who have travelled into countries where horrible executions were to be daily witnessed, for the sake of the excitement which the sight of suffering never fails to give [...] (230)

C'est la jouissance passive du parricide que le texte va s'ingénier à briser, en accumulant la souffrance mais en introduisant une distanciation assez systématique. Au lieu d'une mise en sécurité, le lecteur est confronté à cette logique de la sensation que décrivait Deleuze, et qui ne passe pas par l'excès de la description hyperbolique, mais par la mise en déséquilibre de la posture confortable du lecteur. En effet, le texte déconstruit la posture sadique, semblable à celle du parricide, en engendrant une série de narrations qui se dupliquent à l'infini, enchâssant les niveaux d'écoute, de copie, de lecture. Il devient évident que recopier, écouter ou même se souvenir d'une histoire implique le corps, le met en souffrance, de manière didactique. Ainsi, ce n'est pas la scène de la mort du parricide qui est intéressante en elle-même, mais la manière dont elle est cadrée pour devenir une figure de la violence. Elle est en effet perçue en contre-plongée par Monçada 
qui, dissimulé chez un Juif, devrait rester caché. Or, il est happé par la scène, il cède à la sidération méduséenne de la violence, dans un délire schizophrénique qui le place simultanément en position de victime et de bourreau. C'est en terme de "fascination » qu'il décrit ce qui relève moins de la vision de la scène que de sa lecture, et qui lui arrache des cris : «I echoed the wild shouts of the multitude with a kind of savage instinct [...] then I echoed the screams of the thing that seemed no longer to live, but still could scream » (284). Cet écartèlement relève d'une identification problématique, impossible, et s'adresse directement au lecteur. De même, l'histoire d'horreur met en jeu tout le corps. Dans le souterrain, Monçada se souvient d'une histoire où un homme enflait dans le boyau d'une pyramide, et que ses compagnons souhaitaient dépecer pour qu'il n'obstruât plus le passage : «I could not help recollecting and applying a story I had once read of some travellers who attempted to explore the vaults of the Egyptian pyramids [...] All this detail, that makes so many words to tell, rushed on my soul in a moment;-on my soul? no, on my body. I was all physical feeling, - all intense corporeal agony ». La souffrance annihile tout, absorbe toute humanité, et l'on comprend comment l'on pourrait dévorer sa chair ou celle des autres, « to gnaw out our passage into life and liberty ». Peu importe l'anecdote, ce qui compte ici c'est l'onde de choc, la manière dont l'histoire "s'hystérise ». La matière humaine se distend ou se contracte, dans un espace de vicissitude qui fonctionne par diastole et systole. On peut songer ici encore à Deleuze :

Tout se répartit en diastole et systole répercutées à chaque niveau. La systole, qui serre le corps, et va de la structure à la Figure ; la diastole qui l'étend et le dissipe, de la Figure à la structure. Mais déjà il y a une diastole dans le premier mouvement, quand le corps s'allonge pour mieux s'enfermer; et il y a une systole dans le second mouvement, quand le corps se contracte pour s'échapper. ${ }^{16}$

Ce vers quoi tend le récit, ici, avec son buissonnement d'histoires, c'est bien vers quelque chose qui serait de l'ordre de la sensation plus que de la représentation.

L'appréhension de la violence repose alors non sur les scènes attendues (comme le démembrement du parricide), mais sur la structure démembrée du texte lui-même, basculant d'un épisode à l'autre, continuant à se dévider au moment où le lecteur aspire désespérément à une forme de clôture, de conclusion de l'épisode qui se jouait. Il est difficile de décrire la structure de ce roman, qui s'ouvre ou presque sur un premier décalage, vers le manuscrit de Stanton et le passé, puis se poursuit avec Monçada sauvant le jeune Melmoth (le descendant de Melmoth l'homme errant) de la noyade, pour lui raconter comment, après s'être échappé des prisons de l'Inquisition, il s'est retrouvé chez un juif qui lui a donné à recopier l'histoire de Melmoth et d'Immalee, récit dans lequel Melmoth en personne se met à raconter des histoires de tentations pour persuader le père d'Immalee de rentrer au plus vite afin de la sauver de lui, Melmoth. Puis on passe de la mort d'Immalee à l'Irlande où l'on retrouve comme par miracle à la fois Monçada, Melmoth l'ancêtre et Melmoth le jeune, pour la grande scène finale.

Le texte attire souvent l'attention sur la mise en scène à outrance de la situation narrative, avec les exclamations du jeune Melmoth, et des notes qui nous obligent à des embrayages et débrayages qui dénudent métatextuellement le dispositif, surtout lorsque le narrateur hétérodiégétique peut confirmer que tel ou tel événement a eu lieu à son époque à lui, ou prétend au contraire ne pas savoir de quoi parle Monçada. Le texte décrivant les amours de Melmoth et d'Immalee a une origine à la fois sursaturée et nulle, puisque Adonijah contraint Monçada à recopier la version qu'il a lui-même rédigée, pour tout simplifier, en espagnol transcrit en caractères grecs! Au cœur du souterrain, dans ce 
cabinet de curiosités, les embryons voisinent avec les squelettes qui semblent dicter la réécriture. Or, l'histoire ne dit pas comment Adonijah a pu avoir accès une première fois au texte infernal des amours de Melmoth, et lorsque des pages sont illisibles, Adonijah ne sait absolument pas ce qu'il y avait dedans. Le texte place le lecteur en déséquilibre, en état de frustration, puisqu'il fait se dévider les récits, en refusant à chaque fois la pause consolatrice du dénouement. Ce morcellement a été souligné par de nombreux critiques, comme Victor Sage ou Rosemary Jackson :

Its relentless fragmented structure permits the reader little security. One tale spirals and merges into another, each tale breaking off to lead towards another tale, equally truncated, incomplete. ${ }^{17}$

Jackson parle de boîtes truquées qui n'ont plus de fond. Monçada, lui, emploie la métaphore du chapelet, enfilant les récits. Il me semble que la matière textuelle qui prolifère va plus loin. Il ne s'agit plus ici de narrations gigognes, ni d'impulsion picaresque, mais d'un texte réduit à un corps éviscéré, découpant ses sections en quartiers de chair à vif, dévidant ses entrailles. Le texte se place au-delà de la composition en membra disjecta, de l'outrance, du paroxysme, de l'hyperbole; comme si la mise en crise de la narration, la distanciation délibérée, permettait l'approche asymptotique de l'innommable, de l'irreprésentable, et surtout obligeait le lecteur à se détacher du plaisir du lisant, pour stimuler simultanément le lu et le lectant, opérer une lecture du malaise, de la frustration, qui serait la seule approche possible des figures de la violence.

Il n'est donc pas étonnant que la matière textuelle elle-même, en se dévidant, se troue, s'entaille, résurgence abjecte sécrétée par le passé, le secret. Le premier manuscrit déchiffré par le jeune Melmoth joue à cet égard un rôle emblématique : il est perforé de ponctuation aberrante, de phrases incomplètes, liées à des taches, des excisions, des passages illisibles, jouant non sur l'énigme et l'effet-témoignage gothique, non sur la quête herméneutique, mais sur la simple rupture du texte qui se coupe, manuscrit « discoloured, obliterated and mutilated beyond any that had ever before exercised the patience of a reader » (Melmoth, 32). Plus loin, les fragments de lettres que Juan glisse à son frère Monçada sont maculés, déchirés, illisibles en partie ( from being crushed amid the stones and damp clay contiguous to the door " (194)). L'effet est ironiquement mis en valeur par la mimesis ponctuellement excessive, comme lorsqu'un personnage insignifiant est séduit par un chant et que le narrateur se sent obligé d'inclure la partition de ce chant, ou du moins un fragment, pour le plaisir du lecteur (545) !

Ainsi le texte s'entaille, se troue, va au-delà du dispositif de témoignage propre au roman gothique. La violence ne s'inscrit pas seulement dans la diégèse, c'est le récit qui tente de figurer, par sa structure et ses perforations, une logique de contamination, d'expansion de cette violence qui gangrène l'humain. La structure du texte convie donc à une perception kaléidoscopique des figures de la violence, un peu comme l'enfer selon Bosch nous invite à une lecture simultanée des foyers de souffrance. Dans le vertige de l'oral et de l'écrit, la mise en scène systématique de l'énonciation suggère ironiquement que le propre de la violence, c'est avant tout d'être un scénario qui se répète, que peu importent les détails de la souffrance, les "hiéroglyphes » de la réécriture, seule s'impose cette compulsion de répétition dévoratrice, dans la claustration du tête-à-tête avec la mort.

Si le texte vise à instituer un espace haptique plutôt qu'optique, la fin d'un tel roman devient particulièrement problématique. Tout au long du livre, le récit vise à décentrer la représentation de l'homme errant. Le portrait est brûlé par Melmoth le jeune, et la miniature foulée aux pieds par Monçada. De lui, nous ne voyons que le regard brûlant 
connotant la damnation. Il apparaît toujours aux marges du récit, pas tout à fait à l'incipit, pas tout à fait à la clôture, et sauf dans l'épisode central de la tentation d'Immalee, il ne fait que des apparitions très ponctuelles, en général au moment paroxystique de la souffrance. Il est donc à la fois central et excentré, c'est un être sans individuation, dont nous ne connaissons pas les motivations, ni vraiment l'histoire. Plus que sujet, il devient alors un pur signifiant de la violence errant de récit en récit. Le voyage, malgré l'ubiquité spatiale et temporelle, se fait curieusement immobile, débute et s'achève sur ce roc dominant la mer déchainée, préfigurant les vagues de feu de l'enfer. Mais le principe d'ouverture est préservé par le décalage introduit par le rêve : c'est en songe que Melmoth se voit jeter en enfer, tandis que le livre se clôt sur le jeune Melmoth et Monçada suivant jusqu'à la mer les traces de lutte, cette lutte qui a eu lieu dans l'espace interdit du hors texte. Jusqu'à la fin, Melmoth hante les marges du texte.

La seule figuration possible de la violence, finalement, c'est la réécriture de la vanité réitérée à l'infini sur le mode de la répétition grotesque. D'où l'attitude de Melmoth, contemplant le spectacle du malheur non avec ce pathos voyeuriste de bon aloi, mais en éclatant toujours de rire, "that wild shriek of bitter and convulsive laughter that announces the object of its derision is ourselves " (Melmoth, 354). C'est ce rire qui a fait de Melmoth le héros de Baudelaire :

Melmoth est une contradiction vivante. Il est sorti des conditions fondamentales de la vie ; ses organes ne supportent plus sa pensée. C'est pourquoi ce rire glace et tord les entrailles. C'est un rire qui ne dort jamais, comme une maladie qui va son chemin et exécute un ordre providentiel. Et ainsi le rire de Melmoth, qui est l'expression la plus haute de l'orgueil, accomplit perpétuellement sa fonction, en déchirant et en brûlant les lèvres du rieur irrémissible. ${ }^{18}$

En somme, le rire, c'est la réponse à l'abjection, la ligne de fuite qui constitue à la fois la forme la plus aiguë de la violence et de la souffrance. Le rire, c'est le texte défiguré.

\section{NOTES}

1. Charles Robert Maturin, Melmoth the Wanderer (1820), Harmondsworth, Penguin, 2000, 213.

2. Hubert Teyssandier, Cercles infernaux, Paris, Presses de la Sorbonne Nouvelle, 1992, 75.

3. «[En 1820] un nouveau météore se détache du cadre rituel de la fenêtre ogivale, suspendant son interminable pluie de cendres. » André Breton, Préface (1965), in C.R. Maturin, L'Homme errant, Paris, Phébus, 1996, 15.

4. Gilles Deleuze, Francis Bacon : Logique de la sensation, Paris, La Différence, 1981, 27.

5. Ibid., 29.

6. Voir David Punter, The Literature of Terror, London, Longman, 1996.

7. F.E. Ratchford, W. McCarthy, The Correspondence of Sir Walter Scott and Charles Robert Maturin [1937], New York, Garland, 1980, 59.

8. « [...] in my romance I have determined to display all my diabolical resources, out-Herod all the Herods of the German school ». Ibid., 14.

9. Voir l'introduction de Francis Lacassin au recueil Romans terrifiants, Paris, Laffont, 1984.

10. Philippe Hamon, Expositions, Paris, Corti, 1989, 149. 
11. Yves-Alain Michaux, La Violence, Paris, PUF, 1973, 15.

12. «[...] la visée première d'une question n'est pas, comme on le croit d'ordinaire, d'obtenir une information, mais d'exiger une réponse, d'instaurer un rapport de pouvoir entre questionneur et questionné », Jean-Jacques Lecercle, La Violence du langage (1990), trad. Michèle Garlati, Paris, PUF, 1996, 54-55.

13. Ainsi, le père arrache à son père sénile un quignon de pain, acheté avec l'argent que son propre fils tire de la vente de son sang.

14. Gilles Deleuze, Logique de la sensation, op. cit., 22.

15. René Girard, La Violence et le sacré (1972), Paris, Hachette, 1998, 197.

16. Gilles Deleuze, Logique de la sensation, op. cit., 26.

17. Rosemary Jackson, Fantasy: the Literature of Subversion, London, Routledge, 1981, 104. Voir aussi l'introduction de Victor Sage, dans l'édition utilisée.

18. Charles Baudelaire, (1990), Curiosités esthétiques, et autres cuvres critiques, éd. H. Lemaitre, Paris, Bordas, 1990, 250.

INDEX

oeuvrecitee Melmoth the Wanderer 